\section{Die Mediastinoskopie beim Staging des Bronchialkarzinoms - eine kritische Bewertung ${ }^{1}$}

Zusammenfassung: Hintergrund: Die Mediastinoskopie hat eine hohe Sensitivität für das Lymphknotenstaging und eine Spezifität von $\mathbf{1 0 0} \%$. Der Nachteil der Invasivität ist damit gegenüber bildgebenden Methoden wie CT oder PET gerechtfertigt. Obwohl in vielen Studien Sensitivität, Komplikationen und Kosten erfasst wurden, sind über interindividuelle Abweichungen, bezogen auf den Operateur, keine Daten publiziert. Methode: Zwischen 6/98 und 12/99 wurden in einer prospektiven Untersuchung die biopsierten Lymphknotenstationen erfasst und die histopathologischen Ergebnisse der Mediastinoskopie und nachfolgenden Operation, bezogen auf 6 Operateure, korreliert. Aus den Daten wurde die Sensitivität der Mediastinoskopie für das präoperative Lymphknotenstaging ermittelt. Ergebnisse: Bei 165 Mediastinoskopien betrug die Sensitivität $\mathbf{8 0 , 6 \%}$. Pro Patient wurden 4,1 Lymphknoten biopsiert. Ein erheblicher Unterschied des individuellen Biopsiemusters zwischen Chirurgen wurde bei gleicher operateurspezifischer Sensitivität beobachtet. Die Station 7 wurde in 15,2\%-90,9\% und die Station 2 links in 39,4\%-84,4\% erreicht, während die Station 4 von allen Operateuren einheitlich biopsiert wurde. Schlussfolgerungen: Der Faktor „Operateur“ ist zu vernachlässigen, wenn eine ausreichend große Anzahl von Lymphknotenstationen biopsiert wird. Trotz individueller Biopsietechnik hat die Mediastinoskopie im Vergleich zum CT eine höhere Sensitivität und bleibt Methode der Wahl des präoperativen Stagings. Eine interne Qualitätskontrolle in thoraxchirurgischen Abteilungen ist wünschenswert.

Mediastinoscopy in the Staging of Bronchial Carcinoma A Critical Assessment: Background: Mediastinoscopy has a high sensitivity for lymph node staging with a specificity of $100 \%$. This compensates the disadvantage of being an invasive procedure compared to CT and PET scan. Although many studies examined the sensitivity, rate of complications and costs no data have been published concerning interindividual differences in relation to the surgeon performing the operation. Methods: From 6/98 to $12 / 99$ in a prospective study all biopsied lymph nodes were documented. Histopathologic results of mediastinoscopy and the following operation were correlated with the 6 participating surgeons. From these data the sensitivity of mediastinoscopy for preoperative lymph node staging was analysed. Results: Analysis of 165 mediastinoscopies showed an overall sensitivity of $80.6 \%$. 4.1 lymph nodes were biop-

Pneumologie 2000; 54: 489-493

(c) Georg Thieme Verlag Stuttgart · New York ISSN 0934-8387
G. Leschber, G. Holinka, L. Freitag, A. Linder

Abteilung für Thoraxchirurgie, Lungenklinik Hemer

(Direktor Dr. med. A. Linder) sied per patient. Substantial differences were noted for individual biopsy patterns between surgeons, but surgeon-related sensitivity was similar. Lymph nodes 7 were reached in $15.2 \%$ to $90.9 \%$ and 2 left in $39.4 \%$ to $84.4 \%$, whereas lymph nodes 4 were biopsied at similar rates by all surgeons. Conclusion: The variable "surgeon" can be neglected if a sufficient number of lymph nodes is biopsied. Despite individual biopsy patter mediastinoscopy has a higher sensitivity than CT scan and is still the method for choice of preoperative staging. Internal quality control in thoracic surgery departments is desirable.

\section{Einleitung}

Die Mediastinoskopie gilt noch immer als fester Bestandteil des präoperativen Stagings bei Patienten mit Bronchialkarzinom [1-4] und wurde in die 1988 herausgegebenen „Empfehlungen zur Diagnostik, Stadieneinteilung und operativen Therapie des Bronchialkarzinoms“ der Arbeitsgruppe der Deutschen Gesellschaft für Pneumologie und Tuberkulose und der Deutschen Gesellschaft für Thoraxchirurgie aufgenommen [5].

Die Computertomographie ist als nichtinvasive Staginguntersuchung ebenfalls in diesen Empfehlungen enthalten, erreicht jedoch nicht die hohe Sensitivität und Spezifität der Mediastinoskopie [6]. Die Gefahr des Stagings durch CT allein liegt in der falsch positiven Bewertung vergrößerter mediastinaler Lymphknoten, wodurch man operablen Patienten eine kurative Therapie vorenthalten würde. Mit der PET-Untersuchung wird die in Tumorzellen erhöhte Aktivität des Glukosestoffwechsels erfasst und eine höhere Sensitivität und Spezifität erreicht $[7,8]$. Doch nur die Mediastinoskopie erlaubt die Gewinnung von Gewebe zur histologischen Untersuchung und damit eine Spezifität von 100\%, wogegen die Sensitivität wesentlich vom Umfang der biopsierten mediastinalen Lymphknotenstationen abhängt. Erfahrungen mit diesem Eingriff, der 1959 von Carlens [9] beschrieben wurde, liegen seit langen Jahren vor, ebenso zu dessen Kosten und Nutzen. Wird die Operation von erfahrenen Operateuren durchgeführt, ist die Komplikationsrate gering, und die Belastung für den Patienten ist vertretbar $[10,11]$. Bei einer Staging-Mediastinoskopie werden die ipsi- und kontralateralen mediastinalen

${ }^{1}$ Herrn PD Dr. med. H.-N. Macha zum 60. Geburtstag gewidmet 
Lymphknoten biopsiert oder reseziert und histologisch untersucht. Auf dieser Basis wird das N-Stadium definiert [12]. Das erlaubt dann die stadiengerechte Behandlung des Patienten $[13,14]$. Die Technik der Mediastinoskopie ist zwar hinreichend beschrieben, kann jedoch - da es sich um einen „1Mann-Eingriff“ handelt - nur schwer objektiviert werden. Daher ist nur wenig über das Biopsiemuster verschiedener Operateure bekannt und welche Rolle die Biopsietechnik für die Präzision des N-Stagings spielt.

Die folgende Untersuchung sollte Daten zur internen Qualitätskontrolle der Mediastinoskopie in einer thoraxchirurgischen Abteilung ermitteln und klären, inwieweit der Faktor „Operateur“ die Aussagekraft der Mediastinoskopie beeinflusst.

\section{Patienten und Methoden}

\section{Patientenerfassung und -auswertung}

Zwischen dem 1.6.1998 und 31.12.1999 wurden alle Mediastinoskopien prospektiv erfasst und ausgewertet, insgesamt 366 Eingriffe. In die folgende Auswertung eingeschlossen wurden 165 Patienten, bei denen sowohl mediastinoskopisch als auch operativ gewonnene Lymphknoten zur pathologischen Untersuchung vorlagen. Ausgeschlossen wurden Mediastinoskopien mit rein diagnostischer Intention oder zum Restaging nach neoadjuvanter Therapie.

\section{Erfassungsbogen}

Der Operateur füllte den Erfassungsbogen (s. Abb.1) unmittelbar nach dem Eingriff aus und notierte, wie viele Lymphknoten an den einzelnen Lokalisationen biopsiert oder reseziert wurden. Später wurde anhand der Akten der Patienten die Übereinstimmung des mediastinoskopischen mit dem operativen Lymphknotenstaging geprüft.

\section{Operateure}

Im angegebenen Zeitraum waren sechs Operateure tätig, die zwischen 4 und 44 Eingriffe durchführten. Bis auf einen Chirurgen, der während dieses Zeitraumes in die Mediastinoskopien eingewiesen wurde, hatten die anderen fünf Operateure bereits umfangreiche Erfahrung in der Mediastinoskopie. Ein Chirurg wurde erst im Beobachtungszeitraum in unserer Klinik angestellt, verfügte aber bereits über langjährige thoraxchirurgische Erfahrung.

Technik der Lymphadenektomie bei der Mediastinoskopie und Thorakotomie

Die Nomenklatur der Lymphknotenstationen folgte dem Schema von Naruke [15]. Es war das Ziel jeder Mediastinoskopie, möglichst die Stationen 2 rechts und links, 4 rechts und links sowie $7 \mathrm{zu}$ biopsieren oder Lymphknoten komplett zu resezieren. Lymphknoten anderer Stationen, die im CT vergrößert waren, wurden ebenfalls aufgesucht und biopsiert (Stat. 1 oder 3). Eine erweiterte Mediastinoskopie mit Entnahme der Lymphknoten im aortopulmonalen Fenster oder präaortal wurde nur in Ausnahmefällen durchgeführt.

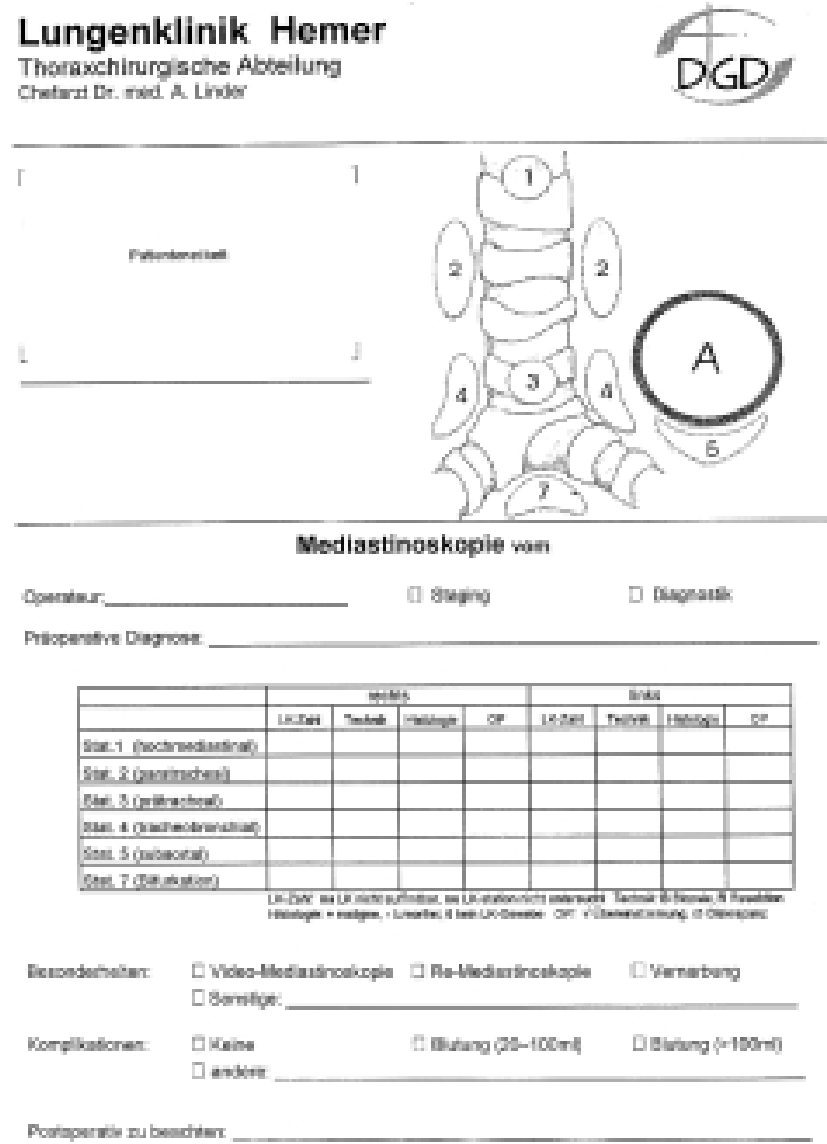

Abb. 1 Der Erfassungsbogen mit den Lymphknotenstationen (Naruke-Schema).

Bei der Operation des Bronchialkarzinoms wurde an die Resektion die systematische Lymphadenektomie angeschlossen. Diese umfasste die Stationen 7, 10, 8 und 9. Bei rechtsseitigen Operationen wurden daneben die Stationen $4 \mathrm{R}$ und $2 \mathrm{R}$ entnommen. Linksseitige Thorakotomien erlaubten außerdem die Entnahme der Lymphknoten der Station 5 und 6 sowie ggf. 4L. Kontralaterale Lymphknoten der Stationen 10 oder 4 wurden entfernt, soweit sie zu erreichen waren. Die Station 7 (subkarinal) ist die einzige, die sowohl bei der Mediastinoskopie als auch bei rechts- und linksseitigen Thorakotomien immer erreichbar ist.

\section{Pathologisch-anatomische Auswertung und Lymphknotenstadiierung nach Mediastinoskopie und Thorakotomie}

Die pathologisch-anatomische Beurteilung erfolgte durch ein der Klinik angeschlossenes pathologisches Institut. Nach makroskopischer Begutachtung wurden dort routinemäßig 4 Schnitte von jeder Lymphknotenstation ausgewertet.

Die histopathologische Stadieneinteilung nach der Operation erfolgte als pN0-pN3, die Stadiierung nach Mediastinoskopie als N0, N2 oder N3, da intrapulmonale Lymphknoten oder solche am Hilus jenseits der Pleuraumschlagfalte (N1) der Mediastinoskopie nicht zugänglich sind. 
Um die Stadiierung vergleichen zu können, wurden bei der Auswertung die operativen Stadien pN0 und pN1 zusammengefasst und mit dem mediastinoskopischen N0 verglichen.

\section{Ergebnisse}

Bei den 165 Staging-Mediastinoskopien wurden durchschnittlich 4,1 Lymphknoten pro Patient erreicht.

Das operative Lymphknotenstadium stimmte mit der mediastinoskopisch ermittelten N-Klassifikation bei 133 der 165 Patienten überein. Daraus resultiert eine Sensitivität von 80,6\% für die Mediastinoskopie. Bei 29 Patienten (17,5\%) stellte sich entgegen dem mediastinoskopisch diagnostizierten N0-Stadium nach der Thorakotomie ein N2-Stadium, bei 3 Patienten ein N3-Stadium heraus (s. Abb. 2).

$\begin{array}{llll}\mathrm{n}=165 & & & \\ \text { Histologie der Operation } & \text { Histologie der Mediastin } \\ & & \mathrm{N} 0 & \mathrm{n}=117 \\ \mathrm{pN} 0 / \mathrm{pN} 1 & \mathrm{n}=117 & \mathrm{~N} 0 & \mathrm{n}=29 \\ \mathrm{pN2} & \mathrm{n}=45 & \mathrm{~N} 2 & \mathrm{n}=16 \\ & & \mathrm{~N} 0 & \mathrm{n}=3\end{array}$

Sensitivität der Mediastinoskopie: 80,6\%

Abb. 2 Übereinstimmung des Lymphknotenstagings der Operation und der Mediastinoskopie.

Die Auswertung der Lymphknoten der Station 7 (subkarinal) ergab eine Biopsierate von 105/165 Patienten (63,6\%). In die Auswertung konnten 91 Patienten einbezogen werden, bei denen sowohl eine Histologie der Mediastinoskopie als auch der Operation vorlag (s. Abb.3). Bei 78 Patienten wurde durch die Mediastinoskopie ein korrektes N-Stadium ermittelt, bei 13 Patienten stellte sich durch die operativ gewonnene Histologie ein höhergradiger pN-Status heraus. Damit beträgt die Sensitivität für die subkarinale Lymphknotenstation 85,7\%.

$$
\begin{array}{llll}
\mathrm{n}=91 & & & \\
& & & \\
\text { Histologie der Operation } & \text { Histologie der Mediastinoskopie } \\
& & \text { N0 } & \mathrm{n}=75 \\
\text { pN0/pN1 } & \mathrm{n}=75 & \text { N0 } & \mathrm{n}=13 \\
\text { pN2 } & \mathrm{n}=16 & \text { N2 } & \mathrm{n}=3
\end{array}
$$

\begin{tabular}{|c|c|c|c|c|c|}
\hline \multicolumn{2}{|c|}{ Operateur } & Stat. 7 & Stat. 4 R & Stat. $2 \mathrm{~L}$ & $\begin{array}{c}\text { Anzahl biopsierter } \\
\text { Lymphknoten }\end{array}$ \\
\hline & $\mathrm{n}$ & $\%$ & $\%$ & $\%$ & $\varnothing \quad(S A)$ \\
\hline A & 33 & 39,4 & 90,9 & 90,9 & $4,2(1,2)$ \\
\hline B & 44 & 45,5 & 97,7 & 68,2 & $4,0 \quad(0,9)$ \\
\hline C & 4 & 75,0 & 75,0 & 25,0 & $3,5(1,3)$ \\
\hline D & 18 & 77,8 & 100,0 & 55,6 & $4,7 \quad(0,8)$ \\
\hline$E$ & 32 & 84,4 & 90,6 & 31,3 & $4,3(1,1)$ \\
\hline$F$ & 33 & 84,8 & 90,9 & 15,2 & $3,7 \quad(1,2)$ \\
\hline$\varnothing$ & 27,3 & 63,6 & 94,5 & 52,1 & $4,1 \quad(1,1)$ \\
\hline
\end{tabular}

\section{Sensitivität der Mediastinoskopie Stat. 7: 85,7\%}

Abb. 3 Übereinstimmung des Lymphknotenstagings der Operation und der Mediastinoskopie bei Lymphknoten der Stat. 7.

Der Vergleich der Häufigkeit, mit der einzelne Lymphknotenlokalisationen biopsiert wurden, zeigt auffällige Unterschiede (Abb.4). Bei der Lymphknotenstation 7 findet man eine
Abb. 4 Häufigkeit der Biopsie bestimmter Lymphknotenstationen der verschiedenen Operateure (SA=Standardabweichung).

aufsteigende Häufigkeit von Operateur A zu Operateur F, wobei $\mathrm{F}$ mehr als doppelt so häufig die subkarinalen Lymphknoten biopsierte als A. Fast reziprok ist dagegen die Entnahmetechnik der Station 2 links. Die tracheobronchialen Lymphknoten auf der rechten Seite wurden von nahezu allen Chirurgen in über $90 \%$ entfernt. Linksseitig wurden die tracheobronchialen Lymphknoten in 82,3\% entnommen.

Die Auswertung der Eingriffe durch die verschiedenen Operateure ergab, dass die Mediastinoskopien sich gleichmäßig auf die einzelnen Chirurgen verteilten. Ausnahmen sind die Kollegen C (in der Anlernphase) und D (Einstellung während des Beobachtungszeitraumes). Im Schnitt wurden 27 Mediastinoskopien pro Operateur durchgeführt. Bei der Anzahl der insgesamt entnommenen Lymphknoten fanden sich signifikante Unterschiede zwischen einzelnen Operateuren (Abb. 5).

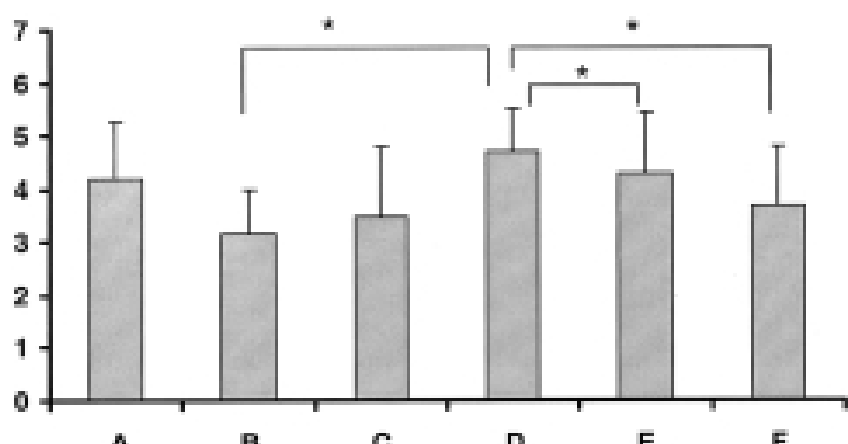

A

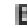

C

Abb. 5 Anzahl der entnommenen Lymphknoten pro Operateur $\left({ }^{*}=p<0,05\right)$.

Komplikationen traten bei Mediastinoskopien nur selten auf. Eine Recurrensparese wurde bei 5 Patienten beobachtet (3,0\%), je einmal bei Operateur A, E und F, zweimal bei Operateur B. Ein signifikanter Unterschied zwischen den Operateuren bestand damit nicht. Einmal kam es zu einer Wundinfektion, die durch lokale Wundbehandlung therapiert wurde. Ein Pneumothorax oder eine Gefäßverletzung, die eine Thorakotomie oder Sternotomie notwendig machten, traten nicht auf. Die Letalität betrug $0,0 \%$. 


\section{Diskussion}

Ein systematisches Problem des Lymphknotenstagings, auch in der vorgestellten Untersuchung, ist die Tatsache, dass Lymphknotenstationen von Ärzten topografisch definiert wurden, dass aber in Wirklichkeit die Grenzen dieser Kompartimente nicht linear verlaufen und fließend sein können. So ist z.B. intraoperativ nur schwer zu definieren, wo die paratracheale Lymphknotenstation (Station 2) endet und die tracheobronchiale (Station 4) beginnt. Ähnlich verhält es sich bei der Abgrenzung prätrachealer von paratrachealen Lymphknoten. Auch ist zu berücksichtigen, dass durch die Längsasymmetrie des Mediastinums eine Verdrängung der von links kommenden Lymphbahnen durch die Aorta aszendens nach rechts erfolgt und somit die „geographische“ Mittellinie mit der topografisch-funktionellen Mittellinie nicht übereinstimmt. Für Therapieentscheidungen ist nur die Differenzierung zwischen rechts und links und damit N2 oder N3 von Bedeutung. Eine kraniokaudale Unsicherheit bezüglich der Zuordnung zu einzelnen Lymphknotenstationen kann vielleicht prognostische Unterschiede erklären, ist jedoch noch ohne Konsequenz für adjuvante oder neoadjuvante Konzepte.

Die Ergebnisse unserer Analyse zeigen, dass die Mediastinoskopie, insbesondere hinsichtlich operateurspezifischer Unterschiede, kritisch gesehen werden muss. Die Biopsiemuster der einzelnen selbständigen Operateure unserer thoraxchirurgischen Abteilung unterschieden sich deutlich bei den Lymphknotenstationen 7 und 2 links. Sie sind am ehesten auf unterschiedliche Ausbildung und individuelle Komplikationserfahrungen zurückzuführen. So birgt die subkarinale Biopsie durch den unmittelbar ventrokranial der Lymphknoten verlaufenden Hauptstamm der Arteria pulmonalis das Risiko einer potenziell letalen Gefäßverletzung. Diese Station wurde nur bei 63,6\% aller Mediastinoskopien erreicht, während die Lymphknoten der Station 4 rechts mit 94,5\% die höchste Biopsierate aufwiesen. Offenbar wird das Risiko der Verletzung der Vena azygos oder der Vena cava geringer eingeschätzt. Die Darstellung der Lymphknoten gelingt hier durch die Orientierung am Tracheobronchialwinkel sicher. Bei Präparation der Station 2 links sind Recurrensparesen zu befürchten, da der Nerv sowohl mechanisch, thermisch als auch elektrisch geschädigt werden kann. Das erklärt, warum diese Lokalisation von einigen Chirurgen seltener biopsiert wurde.

Mit der Mediastinoskopie erreichten wir für das Staging eine Sensitivität von $80,6 \%$. Diese hohe Sensitivität erklärt sich allein dadurch, dass mit der Mediastinoskopie immer mehrere Lymphknotenstationen erreicht wurden. Mit der Biopsie aus nur einer einzigen Station hätte man diese hohe Sensitivität nicht erreicht, denn ein Befall aller ipsilateralen Lymphknotenstationen ist selten. Ebenso selten sind alle Lymphknoten einer Station tumorös infiltriert. Es bleibt also die Forderung nach Biopsie möglichst vieler mediastinaler Lymphknotenstationen.

Werden nur die Lymphknoten der subkarinalen Station betrachtet, erhöht sich die Sensitivität auf 85,6\%. Die höhere individuelle Sensitivität der subkarinalen Lymphknotenstation rechtfertigt jedoch nicht den Schluss, für das präoperative Staging sei nur diese Station zu biopsieren. Denn die Sensitivität von $85,6 \%$ bezieht sich nicht auf alle operierten Patienten, sondern nur auf 105 von 165 Patienten, bei denen verwert- bare Ergebnisse für die subkarinale Station vorlagen. De facto war diese Lymphknotenstation nur in 16/91 Fällen (17,6\%) befallen. Man kann also nicht von einer Schlüsselstation für die lymphogene Metastasierung des Bronchialkarzinoms ausgehen. Dagegen ist es gerechtfertigt, aus unseren Ergebnissen zu schließen, dass der Verlust an Sensitivität gering ist, wenn ausschließlich das apikale Drittel der Lymphknoten der Station 7 erreicht wird, wie dies bei der Mediastinoskopie nur möglich ist. Es liegt nahe anzunehmen, dass die lymphogene Metastasierung in diesem Kompartiment (möglicherweise durch seine Keilform und den Lymphfluss bedingt) einem kranial gerichteten Gradienten unterliegt.

Die globale Sensitivität der Mediastinoskopie ist zwar deutlich besser als die der Computertomographie, deren Sensitivität stark von der Größe der Lymphknoten abhängig ist und zwischen 60-70\% angegeben wird [4,6,14,16,17]. Im Vergleich zu der bei der PET angegebenen Sensitivität von 7096\% [18-20] ist die Überlegenheit dieses operativen Eingriffes jedoch nicht mehr überzeugend. Bereits jetzt vertreten einige Nuklearmediziner die Meinung, dass PET die Mediastinoskopie ersetzen kann [21]. Es ist zu erwarten, dass in Zukunft weniger invasive Methoden wie die Kombination von CT und PET oder die endosonographisch gesteuerte Punktion gleichwertige oder bessere Ergebnisse als die Mediastinoskopie liefern werden. Für die transbronchiale sonographisch kontrollierte Lymphknotenpunktion werden in ersten Untersuchungen Sensitivitäten von 89-100\% angegeben [22-25], wobei die klinische Kontrolle mit Ergebnissen nach intraoperativer systematischer mediastinaler Lymphadenektomie noch aussteht. Auch wird wiederholt auf das deutlich kostengünstigere Vorgehen hingewiesen [26]. Zwar liegen noch keine größeren Studien mit den Komplikationsraten der Endosonographie vor, aber es ist davon auszugehen, dass z. B. Recurrensparesen seltener als bei der Mediastinoskopie vorkommen [27].

In den letzten Jahren ist es zu einer Aufwertung der Mediastinoskopie gekommen, da in den neoadjuvanten Therapieprotokollen des NSCLC das Lymphknotenstaging eine entscheidende Weiche im Behandlungsalgorithmus darstellt. In einigen Studien gilt sie als Vorbedingung zur Einschleusung in das Studienprotokoll $[28,29]$. Inwieweit die neueren Untersuchungsmethoden hier das Vorgehen beeinflussen werden, bleibt abzuwarten.

Die Technik der Mediastinoskopie als „1-Mann-Operation“ konnte in der Vergangenheit nur schwer vermittelt werden. Erst durch die Einführung der Video-Mediastinoskopie ist es möglich geworden, die Methodik einheitlich zu lehren und zu kontrollieren [30]. Die vorgestellten Daten unterstreichen die Bedeutung einer einheitlichen Ausbildung und der internen wie externen Qualitätssicherung der Mediastinoskopie. Nur damit ist erst die Basis für eine Steigerung der Sensitivität dieses immer noch aktuellen Eingriffs geschaffen. 


\section{Literatur}

${ }^{1}$ Ebner H, Marra A, Butturini E, De Santis F. Clinical value of cervical mediastinoscopy in the staging of bronchial carcinoma. Ann Ital Chir 1999; 70: 873-879

${ }^{2}$ Maassen W, Greschuchna D. Die endoskopische und bioptische Untersuchung des Mediastinums. Atemwegs- und Lungenkrankheiten 1975; 3: 161 - 166

${ }^{3}$ Muers MF. Preoperative screening for metastases in lung cancer. Thorax 1994; 49: 1 -2

${ }^{4}$ Pearson FG. Staging of the mediastinum: role of mediastinoscopy and computed tomography. Chest 1993; 103: 346S - 348S

${ }^{5}$ Maassen W, Greschuchna D, Kaiser D, Liebig S, Loddenkemper R, Stapenhorst K, Toomes H. Recommendations on diagnosis, staging, and surgical therapy of lung cancer. Empfehlungen zur Diagnostik, Stadieneinteilung und operativen Therapie des Bronchialkarzinoms. 1988

${ }^{6}$ Takamochi K, Nagai K, Yoshida J, Suzuki K, Ohde Y, Nishimura M, Takahashi K, Nishiwaki Y. The role of computed tomographic scanning in diagnosing mediastinal node involvement in nonsmall cell lung cancer. J Thorac Cardiovasc Surg 2000; 119: $1135-1140$

${ }^{7}$ Scott WJ, Schwabe JL, Gupta NC, Dewan NA, Reeb SD, Sugimoto JT et al. Positron emission tomography of lung tumors and mediastinal lymph nodes using [18 F] Fluorodeoxyglucose. Ann Thorac Surg 1994; 58: 698 - 703

${ }^{8}$ Berlangieri SU, Scott AM. Metabolic staging of lung cancer. N Engl J Med 2000; 343: 290-292

${ }^{9}$ Carlens E. Mediastinoscopy: A method for inspection and tissue biopsy in the superior mediastinum. Dis Chest 1959; 36: $343-$ 352

${ }^{10}$ Hammoud ZT, Anderson RC, Meyers BF, Guthrie TJ, Roper CL, Cooper JD, Patterson GA. The current of mediastinoscopy in the evaluation of thoracic disease. J Thorac Cardiovasc Surg 1999; 118: $894-899$

${ }^{11}$ Luke WP, Pearson FG, Todd TRJ, Patterson GA, Cooper JD. Prospective evaluation of mediastinoscopy for assessment of carcinoma of the lung. J Thorac Cardiovasc Surg 1986; 91: 53 56

12 Dienemann H, Hoffmann H, Koebe HG. Technik und Rationale der Lymphknotendissektion bei Bronchialcarcinom. Chirurg 1998; 69: $412-417$

${ }^{13}$ Böhle AS, Kurdow R, Dohrmann P. Rationelle Diagnostik des Bronchialkarzinoms. Dtsch med Wschr 1999; 124: 1137-1141

${ }^{14}$ De Leyn P, Vansteenkiste J, Cuypers P, Deneffe G, Van Raemdonck $\mathrm{D}$, Coosemans W, Verschakelen J, Lerut $\mathrm{T}$. Role of cervical mediastinscopy in staging of non-small cell lung cancer without enlarged mediastinal lymph nodes on CT scan. Eur J Cardiothorac Surg 1997; 12: 706-712

${ }^{15}$ Naruke T, Goya T, Tsuchiya R, Suemasu K. Prognosis and survival in resected lung carcinoma based on the new international staging system. J Thorac Cardiovasc Surg 1988; 96: 440-447

${ }^{16}$ Gdeedo A, Van Schil P, Corthouts B, Van Mieghem F, Van Meerbeck J, Van Marck E. Prospective evaluation of computed tomography and mediastinoscopy in mediastinal lymph node staging. Eur Respir J 1997; 10: 1547 - 1551

${ }^{17}$ Dillemans B, Deneffe G, Verschakelen J, Decramer M. Value of computed tomography and mediastinoscopy in treoperative evaluation of mediastinal nodes in non-small cell lung cancer. A study of 569 patients. Eur J Cardiothorac Surg 1994; 8: 37-42

18 Pietermann RM, Van Putten JWG, Meuzelaar JJ, Mooyaart EL, Vaalburg W, Koëter GH, Fidler V, Pruim J, Groen HJM. Preoperative staging of non-small-cell lung cancer with positron-emission tomography. N Eng J Med 2000; 343: 254-261

${ }^{19}$ Gupta NC, Graeber GM, Bishop HA. Comparative efficacy of positron emission tomography with fluorodeoxyglucose in eva- luation of small $(<1 \mathrm{~cm})$, intermediate $(1$ to $3 \mathrm{~cm})$ and large $(>3 \mathrm{~cm}$ ) lymph node lesions. Chest 2000; 117: 773-778

${ }^{20}$ Scott WJ, Gobar LS, Terry JD, Dewan NA, Sunderland JJ. Mediastinal lymph node staging of non-small-cell lung cancer: a prospective comparison of computed tomography and positron emission tomography. J Thorac Cardiovasc Surg 1996; 111: 642 648

${ }^{21}$ Stokkel MP, Bakker PF, Heine R, Schlosser NJ, Lammers JW, Van Rijk PP. Staging of lymph nodes with FDG dual-headed PET in patients with non-small-cell lung cancer. Nucl Med Commun 1999; 20: 1001 - 1007

22 Serna DL, Aryan HE, Chang KJ, Brenner M, Tran LM, Chen JC. An early comparison between endoscopic ultrasound-guided fineneedle aspiration and mediastinoscopy for diagnosis of mediastinal malignancy. Am Surg 1998; 64: 1014-1018

${ }^{23}$ Fritscher-Ravens A, Pforte A. Endosonographisch gesteuerte Feinnadelaspirationsbiopsie. Dt Ärzteblatt 1999; 96: B2402B2406

${ }^{24}$ Janssen J, Johanns W, Luis W, Greiner L. [Clinica value of endoscopic ultrasound-guided transesophageal fine needle puncture of mediastinal lesions]. Dtsch Med Wschr 1998; 123 : $1402-1409$

${ }^{25}$ Huenerbein M, Ghadimi BM, Haensch W, Schlag PM. Transesophageal biopsy of mediastinal and pulmonary tumors by means of endoscopic ultrasound guidance. J Thorac Cardiovasc Surg 1998; 116: $554-559$

${ }^{26}$ Aabakken L, Silvestri GA, Hawes R, Reed CE, Marsi V, Hoffmann B. Cost-efficacy of endoscopic ultrasonography with fine-needle aspiration vs. mediastinotomy in patients with lung cancer with suspected mediastinal adenopathy. Endoscopy 1999; 31: $707-$ 711

${ }^{27}$ Bonadies J, D'Agostino RS, Ruskis AF, Ponn RB. Outpatient mediastinoscopy. J Thorac Cardiovasc Surg 1993; 106: 686-688

${ }^{28}$ Eberhardt W, Wilke H, Stamatis G, Stuschke M, Harstrick A, Menker H, Krause B, Müller MR, Stahl M, Flasshove M, Budach V, Greschuchna D, Konietzko N, Sack H, Seeber S. Preoperative chemotherapy followed by concurrent chemoradiation therapy based on hyperfractionated accelerated radiotherapy and definitive surgery in locally advanced non-small-cell lung cancer: mature results of a phase II trial. J Clin Oncol 1998; 16: 622-634

${ }^{29}$ Thomas M, Rübe C, Semik M, von Eiff M, Freitag L, Macha HN, Wagner W, Klinke F, Scheld HH, Willich N, Berdel WE, Junker K. Impact of preoperative bimodality induction including twice daily radiation on tumor regression and survival in stage III nonsmall-cell lung cancer. J Clin Oncol 1999; 17: 1185

${ }^{30}$ Hürtgen M, Friedel G, Kyriss T, Toomes H, Linder A, Fritz P. Radical video-assisted mediastinoscopic lymphadenectomy (VAMLA) - technique and first results. Eingereicht beim European Journal of Cardio-Thoracic Surgery

\section{Dr. med. G. Leschber}

Abt. f. Thoraxchirurgie

Lungenklinik Hemer

Theo-Funccius-Str. 1

58675 Hemer 